\title{
The role of data-driven artificial intelligence on COVID-19 disease management in public sphere: a review
}

\author{
Sini V. Pillai $\mathbb{B}$ - Ranjith S. Kumar
}

Accepted: 9 September 2021/Published online: 30 September 2021

(C) Indian Institute of Management Calcutta 2021

\begin{abstract}
Coronavirus disease 2019 (COVID-19) is an infectious disease with acute intense respiratory syndrome which spread around the world for the very first time impacting the way of life with drastic uncertainty. It rapidly reached almost every nook and corner of the world and the World Health Organization (WHO) has announced COVID-19 as a pandemic. The health care institutions around the globe are looking for viable and real-time technological solutions to handle the virus for evading its spread and circumvent probable demises. Importantly, the artificial intelligence tools and techniques are playing a major role in fighting the effect of virus on the economic jolt by mimicking human intelligence by screening, analyzing, predicting and tracking the existing and likely future patients. Since the first reported case, all the government organizations in the world jumped into action to prevent it and many studies reported the role of $\mathrm{AI}$ in taking decisions analyzing big data available in public sphere. Thereby, this review focuses on identifying the significant implication of AI
\end{abstract}

S. V. Pillai $(\bowtie)$

College of Engineering Trivandrum, CET School of Management, Thiruvananthapuram, Kerala 695016, India e-mail: sini.mba@cet.ac.in

\section{R. S. Kumar}

Micro/Nanofluidics Research Laboratory, Department of Mechanical Engineering, College of Engineering Trivandrum, Thiruvananthapuram, Kerala 695016, India e-mail: ranjith@cet.ac.in techniques used for the COVID-19 disease management in the public sphere by agglomerating the latest available information. It also discusses the pitfalls and future directions in handling sensitive big data required for advanced neural networks.

Keywords Data analytics · Data-driven decision · Artificial intelligence $\cdot$ Deep learning $\cdot$ Machine learning

\section{Introduction}

Artificial intelligence (AI) as a tool is echoing towering hopes to tame the viral pandemic that has extensively affected the entire world since November 2019. The SARS-CoV 2 aliases Coronavirus disease 2019 (COVID-19) is one of the deadly RNA viruses that has brought the entire world and humanity to a standstill since the beginning of the year 2020. Indeed, no one including scientists, healthcare professionals, technicians, financial advisors or engineers was able to predict the outbreak or take precautions a priori. Interestingly, the application of AI was visible in the beginning of the dissemination of COVID-19 (Hsiang et al. 2020), a Canadian startup company BlueDot was the first to raise an alarm regarding the upsurge of a communicable respiratory disease even before the official announcement of COVID-19 by the authorities 
(Bobdey and Ray 2020). This was actually raised by AI researchers who employed advanced AI techniques like deep learning (DL) and machine learning (ML) by collecting data from various social media chats. As per the reports released by the WHO, AI and big data are an integral part of the successful response to the novel corona disease in China. Many research works highlight the adoption of advanced digital technology tools for surveillance, prediction and diagnosis to fight the COVID-19 pandemic (Golinelli et al. 2020). Thereby, AI can play a crucial role in ensuring policies, management and resource allocation to generate sustainable solutions to the most inversely impacted by the COVID-19 pandemic (Taylor 2020).

Every industry is struggling to survive and at the same time giving out every ounce of their efforts toward the fight against this catastrophe. Generally, an early and prompt detection will reduce and keep in track the spread of the disease and provide sufficient time for the health care experts to save more lives by an early diagnosis. The AI technology has come into view which could possibly be significant in restricting the spread of the virus within limits (Hui et al. 2019). David Heymann, Executive Director of the Communicable Diseases Cluster, WHO, explained various important elements to be looked into for the successful implementation of public health response during the outbreak of a new disease. The factors include, gravity of transmissibility among risked or vulnerable population, history of infection, incubation period and mortality rate, identifying and characterizing the causative organism as well as epidemiological modeling to suggest effective prevention and control measures. In this regard, AI can make a major contribution to the current outbreak and reduce the burden on clinicians by expediting the diagnoses as well as prognosis (McCall 2020). To bring significant changes in the health care requires up gradation in patient care, follow up, treatment and anticipating the patients who are more likely to get severe disease. Making use of health records and medical imagery together helps in predicting results of the patients which will further be helpful for estimating the need of more healthcare professionals, assistants, medicines and equipment in future (Luengo-Oroz et al. 2020).

Early screening and diagnosis are very crucial to identify the affected patients and to control its spread. Here, AI in general and ML in particular have significantly improved prediction, forecasting, contact tracing for the COVID-19 pandemic and has reduced the human intervention in medical practice and the models deployed are up to the mark to tackle the SARS-CoV-2 epidemic (Lalmuanawma et al. 2020). Essentially, AI aids in collecting all the necessary information from institutions or people who are exposed to the disease sites and can be miraculously utilized by examining information from evidence of events during the outbreak.

A comprehensive and compact monitoring of social media can provide many pointers on further detailed interrogation. The past travel information can speak a lot about people who have visited the outbreak places and keep them on a watch. The virus has spread across many other countries in no time and these countries have set up extremely good systems of contact-tracing and patient isolation. The AI finds its application in contact tracing, IR imaging, surveillance, identifying social distancing measures and effectiveness, face mask detection and also uses data from social media to fight against the pandemic, see Fig. 1. Most importantly, substantial amount of data and its classification were generated during these difficult times in multiple fronts and can be useful in identifying relevant patterns out of it. In connection with that, the present review emphasizes the pivotal role played by datadriven $\mathrm{AI}$ in the containment of COVID-19 disease in the public sphere. A review of existing literature on AI in COVID-19 management especially focusing on available reviews is given below.

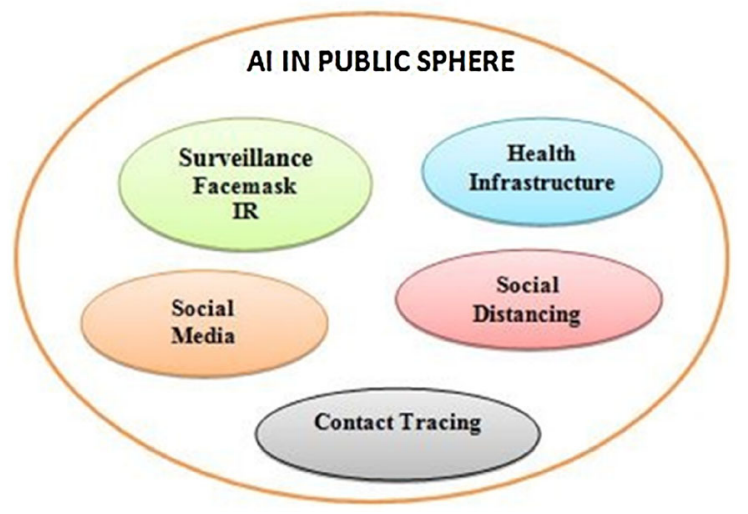

Fig. 1 Schematic diagram representing implications of AI in containing COVID-19 spread in public sphere 


\section{Review of literature}

Digital technologies have been reported in various literature published especially in PubMed and MedrXiv with a potential to fight against the COVID-19 pandemic after its inception in November 2019. Here, artificial intelligence-powered tools are found effective for prevention, surveillance, diagnosis and screening of COVID-19 disease (Golinelli et al. 2020; Beard and Belchamber 2020). The versatility of artificial intelligence supports the efforts to address the biomedical, epidemiological, and socio-economic challenges to combat COVID-19 globally (Leslie 2020). It is noticed that the data-driven AI implementations are subsequently making a paradigm shift in health care industry with respect to the current COVID-19 outbreak even by predicting the location of the next outbreak (McCall 2020; Chen and See 2020; Mohanty et al. 2020). Interestingly, advanced AI models find applications to diagnose illness from imagery, predict patient outcomes, and filter misinformation and disinformation based on spreading patterns through social networks. Moreover, in future more effective AI governance frameworks will be required to take into account ethical, social, clinical, legal and commercial considerations (Bullock et al. 2020; Chamola et al. 2020; Shi et al. 2020). The human interventions in medical practice have significantly reduced as AI applications extensively improved treatment, medication, screening, prediction, forecasting, contact tracing, and drug/vaccine development process for the COVID-19 pandemic (Lalmuanawma et al. 2020; Leslie 2020; Fang et al. 2019; Vaishya et al. 2020; Lou et al. 2020; Mohamadou et al. 2020; Kim 2020). Ever since the outbreak initiated worldwide, every affected country is looking into the significance of the right forecast to restrict the spread of coronavirus. On the other hand, most of the models are not deployed to illustrate their real-world operation, but are still up to the spot to deal with the SARS-CoV-2 epidemic. Though these surveys elaborate on how AI deals with the COVID-19 control in many fronts, an exclusive review on application of AI utilizing the big data generated in the public sphere is elusive.

\section{Disease spread prevention in public sphere}

To check the exponential progression of the disease the stakeholders adopted different AI-based strategies especially in public sphere. Here, the major domains identified are healthcare, surveillance, contact tracing, social distancing, social media and industry applications. Following sections briefly discuss how the big data analysis using AI drive the decision making to control the repercussions in the society owing to the novel coronavirus.

\section{Health infrastructure}

Today, the medical field is a data-driven domain due to the increased utilization of electronic medical records. Consequently, AI has significant impact in the medical field employing machine learning and similar advanced techniques (Schwartz et al. 2019). Moreover, it is important to learn from the data and create discovery-support systems to enable the human physician to identify vulnerable populations. For instance, China, which is having strong foundation in technological advancements, has already tapped the advantage of AI to forecast the evolution of disease outbreaks, to speed up genome sequencing, enhance diagnosis and also to deploy robots for treatment. AIbased systems have a positive impact on various diagnostic fields such as radiology, pathology, dermatology, and ophthalmology and are expected to increase the diagnostic efficiency (Hsieh 2017; Pearl 2018). Additionally, AI-based systems have greater opportunities in robotic surgery and a surgeon has an opportunity to carry out the procedures with telepresence (Wottawa et al. 2016). The advances in computing power, learning algorithms and the availability of large datasets from digital medical records and wearable health surveillance devices constructs its position appealing in medicine and healthcare.

In addition, AI technologies strengthen the power of medical imaging tools which are used in frontline hospitals through the integration with X-ray and computed tomography (CT) for effective rapid diagnosis in the fight against COVID-19 to make clinical decisions for tracking and diagnosis (Shi et al. 2020). Moreover, AI has been used to analyze symptoms of pneumonia which has a high possibility of getting COVID-19 by detecting it with the help of CT scans (Simonite 2020). The technique helps in 
understanding symptoms and signs of COVID-19 pneumonia and the medical staff can proceed with further examinations to detect the presence of the disease. The performance of radiology images like $\mathrm{X}$-ray and CT scans seems to fail during the high flareup of the pandemic. A manual CT scan can take 15 minutes, but AI can read the image in 10 seconds and make fast judgments. Artificial intelligence (AI) systems are capable to precisely differentiate COVID-19 infections (Shuja et al. 2020). Research has been done integrating $\mathrm{AI}$ and ML on $1020 \mathrm{CT}$ images of 108 infected patients including viral pneumonia of 86 patients recommends the application of the convolutional neural network as a suitable tool for radiologists (Lalmuanawma et al. 2020).

Fudan University and Shanghai city government developed a unique AI-based medical check-up for respiratory blockage issues and tested on more than 93\% of Shanghai residents (Shaw et al. 2020). The system proved to have a potential to perform quick diagnosis when the health system is clogged (Yan et al. 2020). It is also found that the statistical tests on full blood count using AI-assisted machine learning could improve initial screening for patients at an early stage of infection and can be distinguished from other viral infections (Banerjee et al. 2020). Furthermore, it is highly imperative to identify patients with symptoms like fever and cough in a minimum period of time from low and middle income countries which have resource limited settings. Techniques of deep learning can help in identifying patients prone to COVID-19 from patterns in X-ray images and other CT scans and AI systems installed in various functional areas have helped in reducing the time required to review the scans (Luengo-Oroz et al. 2020). The technology available in most hospitals will be sufficient to identify COVID-19 infections through extraction and subsequent classification of deep features from chest X-ray images using machine learning methods (Al-Timemy et al. 2020). Note that, important aspects with regard to guidelines for clinical trials evaluating interventions with an AI component to identify impact on health outcomes have to comply with consolidated standards of reporting trials-artificial intelligence (CONSORT$\mathrm{AI})$ and standard protocol items: recommendations for interventional trials-artificial intelligence (SPIRITAI). Here, CONSORT-AI promotes transparency in reporting clinical trials for $\mathrm{AI}$ interventions and critically appraises the quality of clinical trial design and risk of bias in the reported health outcomes (Liu et al. 2020; Rivera et al. 2020). Another important aspect that can be handled by AI techniques using such big data is the disease forecasting, indeed that could predict the spread of viruses effectively for early interventions (Mohamadou et al. 2020; Raza 2020; Hu et al. 2020). For instance, an AI model using linear regression, multi-layer perceptron and vector autoregression method can solve the problems raised by COVID-19 by predicting the proliferation of virus and anticipating the growth pace of COVID-19 cases in India (Sujath et al. 2020). In similar lines, the Random-Forest scheme which uses the COVID-19 patient's demographical; travel and health data forecasts the recovery rate with an accuracy of $94 \%$ (Iwendi et al. 2020). Moreover, COVID-19 mortality prediction and estimation of future death cases in Indian context is developed with statistical neural network (SNN) models with hybrid version to consider error variables using probabilistic radial basis function and generalized regression neural networks. The obtained results will facilitate the public health officials for better prevention and control measures for COVID-19 in a time-bound manner (Dhamodharavadhani et al. 2020).

Currently, the market share of AI is $40 \%$ and is anticipated to reach $\$ 6.6$ billion by 2021 and estimates that $\mathrm{AI}$ has the potential to improve patient outcomes by $30-40 \%$ while reducing treatment costs to $50 \%$ (He et al. 2019). Artificial intelligence is a revolutionary concept in healthcare and can transform the clinical practices of physicians across the world in formulating better clinical decision making (Emanuel and Wachter 2019). The prediction of the structure of virus through protein folding experimentation has hustled up with the support of AI. Further, the spread of the virus is explored with an AI supported algorithm (Jakhar and Kaur 2020). Wearable healthcare devices which are basically the Internet-of-things (IoT) devices can be integrated with AI so that the monitoring and surveillance become more efficient by utilizing the huge dataset created by the user-end sensors (Allam and Jones 2020). The wearable devices can record the patient's heart rate, breathing capacity and body temperature. A fitness tracker can record the blood pressure and insulin monitors will enable the healthcare system by directly feeding data into larger AIassisted analytic networks (Jamal et al. 2018). 
The AI can better understand the COVID-19 crisis and bring one step closer to averting the pandemic by identifying patterns and providing positive insights (Dwivedi et al. 2020). The scientists, researchers, health experts and clinicians were working with new technologies to flatten the curve and tackle the pandemic. AI can also be used to develop AI-powered Chatbots (Maniou and Veglis 2020) to expand the communication channel to the public and give advice regarding COVID-19 treatment and guidelines. The Chatbots can actually call in many individuals at once rather than a manned call contacting and advising one person at a time. Further, the AI tools can be used in screening methods to deploy the integrative medicine techniques to fight against COVID-19 (Ahuja et al. 2020). The chats were about people having fever and respiratory distress and the biggest challenge was to process the data in an effective manner obtained from different sources in different forms (Bobdey and Ray 2020).

A new model for screening with automatic COVID19 detection based on deep learning algorithms could help in screening the patients at the earliest and helps in rapid diagnosis with enhanced accuracy (Lalmuanawma et al. 2020). Neural networks are developed to extract the virus spread features and help in analyzing day-to-day updates of the patients (Vaishya et al. 2020). Researchers from the artificial intelligence arena have been emphasized on developing better methods to examine this situation with the help of data mining. Here, machine learning and deep learning models are utilized to forecast the future damages of COVID-19 globally with the real-time information and data (Punn et al. 2020). In the similar lines, the researchers at the Chan Zuckerberg Biohub in California have built a model to estimate the number of COVID-19 infections that have been undetected and the consequences for public health analyzing twelve regions across the globe ( $\mathrm{Li}$ and Ayscue 2020).

Several companies are using AI technologies to improve their existing drugs as well as to develop novel drugs to treat the COVID-19 (Mohanty et al. 2020). Alex Zhavoronkov, CEO of Insilico Medicine, believes AI can act as a catalyst to all the operations in a cheaper, faster, and error free manner in order to accomplish the desired outcome. Hong Kong-based Insilico Medicine also generated novel molecules with the potential to bind a specific SARS-CoV-2 protein through an AI-based drug discovery platform. The molecule can block the virus's ability to replicate. SRI International and AI company Iktos announced that they are collaborating together to develop new antiviral therapies to treat COVID-19 (Kumar and Sharma 2020). The Researchers of Huazhong University of Science and Technology and Tongji Hospital in Wuhan developed XGBoost machine learning-based prognostic model which helps in diagnosing COVID19 using blood samples with 90\% accuracy (Dananjayan and Raj 2020). Also a diagnostic tool to distinguish COVID-19 from other pneumonia has also been developed in Wuhan. The most modern equipped medical center Zhongnan Hospital, Wuhan University, China, became a trial for being adaptive to a new infectious disease which has the possibility of becoming a pandemic. In order to accelerate the research process very large datasets in epidemiology, bioinformatics and molecular modeling, for this purpose required computing power were given to the authorities by IBM, Amazon, Google and Microsoft (Lardinois 2020).

Every country has had its own share of trials and is extensively adapting to the increasing need for urgent health care facilities. Many researchers have pointed out the increasing anxiety, loneliness, and depression even leading to mental illness among the people in quarantine both homes and in hospitals. Very basic e-health tools might be handy in the situation to create a communication between them which will create a positive thought of someone keeping a check on them, which induces more quality of life and speedy recovery. Center of e-health providers have initiated to give a global outline of all the e-health solutions possible for coping up with the COVID-19 through telemedicine (Pappot et al. 2020). Typically, electronic health records help the health workers to record disease history of patients, so that the overall management is improved by providing quick exchange of information, keeping track of the spread of disease, assisting in virtual consultation as well (Reeves et al. 2020). The services can be utilized for better communication between patients and hospital authorities and at the same time e-health can be used for obtaining patients' views to acquire the e-health solutions for the overall betterment. Furthermore, the introduction of telemedicine has helped the healthcare professionals to give out their time to cases with utmost preferences by sitting in a remote safe location (Luengo-Oroz et al. 2020). 
A unique health barcode system with three color coding system can identify the affected people and also inform whether people have been in proximity to any infected public through online mapping. The color green represents good health, yellow for caution and red for infected people. Career's big data with Baidu's location data formed the input to develop the health barcode (Shaw et al. 2020) in Wuhan from the second week of February 2020.

\section{Surveillance}

Strict social distancing and close monitoring are the most effective strategies for the foreseeable future with regard to spike in COVID-19 cases. Participatory disease surveillance mechanisms (Garg et al. 2020) have already been adopted in numerous countries. In India, with limited resources, telecom industry and the healthcare system makes the way for initiating participatory disease surveillance. The Aarogya Setu, an application developed by India, as part of the disease surveillance initiative in India gives authentic information on time using a hybrid of Bluetooth and location generated social graph and is easily accessible to all (Garg et al. 2020). All the necessary verified information is available and the public can take precautions based on the information. A new AI model, sentiment informed time series analyzing AI (SITALA) has been conditioned on COVID-19 positivity data to focus on forecasts that restrict the spread of COVID-19 in Houston, one of the big hotspot (infection centers) of the virus outbreak in the US (Desai 2020).

The location tracking of the infected persons was done using Bluetooth, GPS, social graph, contact details, digital card transaction data and systematic physical address (Garofalo et al. 2020). AI-based techniques help in identifying the clusters and hotspots and also assist in tracing contact of infected individuals using mobile applications. Further the prediction of most vulnerable regions and number of cases/death in these regions can also be predicted. It also predicts the likely reappearance of the virus. A national geographic information system (GIS)-based health infrastructure can be of help to deal with epidemics and pandemics through mapping the hotspots (Ranga et al. 2020). Google maps and Bing maps can be used to organize data to get a clear picture of the most affected places which are widely accessible to the
Government and citizens along with a range of services including health care, food, essential services to day-to-day life services. To effectively bring it into life, the Government needs to enforce a mandatory submission of goods and services tax service providers. Once the stage is set for this to initiate, the Government can effectively control the timing of services accordingly. Also authentic information can be passed to citizens in no time. Chinese authorities were able to track down people who had visited Wuhan market, with the help of migration maps (Whitelaw et al. 2020), which use mobile phones, mobile payment applications, and social media to collect data about location of people who visited the market. Similarly Taiwan had enquired the list of travelers who had visited Wuhan by inferring about the details from immigration records and airline companies. Globally in the wake of the pandemic nations is rapidly developing trustworthy informatics infrastructure in a world where there is an alarmingly increasing rate of COVID-19 patients. The health infrastructure system shall help in management of the pandemic more effectively. Every nation needs to unite in understanding more about the opportunities in artificial intelligence and work toward the overall safety and security as AI will be the future technology of the world.

A detection and tracking surveillance system using IP surveillance and thermal camera can identify, locate, and monitor the movement of confirmed COVID-19 cases, track primary contacts, identify high risk infected areas, monitor social distancing, body temperature detection and detection of mask wearing (Hossain et al. 2020). The captured signals are used to run the deep learning model to take remedial actions accordingly. The COVID-19 vulnerability index, 'C-19 Index' is being used by healthcare systems for care management organizations and insurance companies to identify high-risk individuals, further informing them about the importance of handwashing and social distancing and also offering to deliver food, toilet paper and other essential supplies so they can stay indoors (Reps et al. 2020).

Another important aspect in surveillance is the application of fifth generation (5G) communication technology in combination with different other technologies especially in logistics. 5G technology with drones was used in the transport system to identify infringement of laws, with thermal cameras for quick 
thermal screening of people to communicate the date and time details, with robotics for city sanitization. Furthermore, 5G communication technology was also used for tele-medical care (Bullock et al. 2020).

The viral spread is too fast to be contained by manual contact tracing, but can be controlled with a faster process. Based on data published as of 29th of March 2020, more than 30,000 deaths were reported and more than 23,000 people are in critical conditions globally affecting more than 650,000 people (Shaw et al. 2020). The virus outbreak is happening in a digitized and more connected world making data collection much easier. Advanced computational models with digital data via machine learning proved the potential of handling COVID-19 outbreak. Extensive use of emerging technologies along with the health care treatment will make the response more effective and reduce risk of spread of the disease. Artificial intelligence, big data, 5G technologies were used in combination with other emerging technologies like drones, automated vehicles, block chain and robotics (Shaw et al. 2020) to play a significant role in controlling the impact during the four stages of the pandemic outbreak such as impacted case only, local transmission, clusters of cases, community transmission. The duration and impact of each stage is critical and the final stage of the pandemic can only be managed with a vaccine which has to be approved by WHO. Population dominated countries are now inducted into the fourth stage (Chamola et al. 2020) experimenting on IoT, robots, smart wearables, AI, block chain and $5 \mathrm{G}$ to control the outbreak effectively

COVID-19 cases are popping up every day and leaving everything necessary to be monitored by the Government uninterruptedly. The identified methods to restrain from the spread of the virus are by practicing social distancing, wearing masks in public places and maintaining hygiene throughout. To detect if people strictly follow the new normal, Bangalorebased Integration Wizards Solution came up with a solution to monitor social distancing and detect the usage of face mask. The algorithm has been trained to track these and has also included thermal screening. For mask detecting, the Viola-Jones face detection algorithm is used to extract the features from the face area using the deep neural network (Hossain et al. 2020). Similarly, the public interest in wearing face masks as a protection toward COVID-19 epidemic is done using Google Trends relative search volume
(RSV) data generated by an AI backbone. Note that, it explored whether early awareness on face mask use could help contain the outbreak effectively. The trend observed a divergent pattern of RSV values with some geographical regions having peak RSV values early in the epidemic and correlated with the epidemics in different regions. The results suggested that early public interest with face mask will be an independently important factor in controlling the COVID-19 epidemic on a population scale and mitigate the current pandemic (Wong et al. 2020).

The most recent contribution by the startup, IRIS, makes use of deep integration with hardware which in turn permits the system to run efficiently. Its software converts legacy applications to work on modern IoT and mobility standards, and uses data to make business teams use assets efficiently. From the live videos, AI technology makes use of deep neural networks to process data to detect and identify and monitor face masks, personal protective equipment (PPE), people and social distancing. Emails or Whatsapp messages will be sent to concerned authorities on noncompliance of the safety precautions. Since the world is slowly lifting up the restrictions companies are resorting to innovations in this area to ensure safety of their workforce. Hence the possibility of the spread of virus can be monitored and minimized to an extent through this technique in public space.

\section{Contact tracing}

Most modern technologies and advancements in AI and machine learning have been a great source in tracing and predicting the prospective infectious people and various other diseases (Ienca and Vayena 2020). Mobile technologies like GPS, Bluetooth, cellphone masts with the support of AI-powered big data analytics seems to be in the forefront in the fight against COVID-19 managing the spread of the virus. Typically, the rate of spread of the virus and people to people transmission is high due to higher mobility of people, contact tracing is difficult but very helpful in this context. Personal information as mobile-based data can solve the problem of identifying the people in quarantine and making a region into a containment zone.

For instance, China, the most populated country, made use of data from institutions across the country and various other health applications to control the 
disease spread (Ienca and Vayena 2020). Alipay Health Code, an Alibaba backed application run by the Government supported decisions about who should be quarantined for COVID-19 after examining the data. Note that, Baidu big data was used to identify clusters of infected people and was an effective COVID-19 management tool in China (Shaw et al. 2020). Data-driven applications speed up contact tracing at individual levels to target health surveillance as well as to enable ease of personalized approaches to social distancing measures (Leslie 2020). During this unprecedented time, Aarogya Setu app has been phenomenal in giving people information about the propagation of the deadly coronavirus in India. The Government is taking every step to give early warnings and precautions to citizens, providing timely alerts and information, which further helps to tame the spread of COVID-19. The major boon of the app is it gives out only authentic and verified information. Similarly Singapore government has implemented a Bluetooth-based proximity tracing system, TraceTogether that helps public health officials to track down and communicate with the at-risk contacts of infected users (Bay et al. 2020).

The data stored in mobile phones gives a lot of information about the whereabouts of people; however accessing the information of a person without consent may lead to violation of personal rights and as violation of privacy laws (Ienca and Vayena 2020). Indeed, collection of large-scale public data is intruding into the privacy of the public to some extent. However, the data are used in protected manner by the countries across the world preserving personal privacy while leveraging mobile computing data to potentially save lives. However, not complying with the privacy guidelines may lead to loss of public trust in the authorities. As data is an inevitable factor in controlling the mass outbreak in the modern digital era, collecting and pooling of information from affected people should be done with utmost security. The data collection should be done so as to achieve a specific requirement of the health objective and the purpose for tracking the electronic devices should be made only for health related matters. The societal interventions like digital contact tracing are raising fears among the community and are challenging the commitments to privacy matters (Leslie 2020). Huge amount of time and effort have been dedicated toward making use of digital technology to make the system more automated, as the majority have access to smartphones with integrated location tracking facility. This whole contact tracing process is at the stake of privacy constraints. There is always a dilemma between data first or privacy first issues. Mutually initiated data processing agreements can solve the digital data problem up to certain extent.

\section{Social distancing}

Based on all the studies, it is identified that strong social distancing measures are the most operational and effective method of preventing the containment of virus in the public sphere. As social distancing is the major way to contain the spread of virus and bring the pandemic under control, it is of higher priority to check if the new norms of social distancing are abided (Courtemanche et al. 2020). Inspired by Andrew Ng's AI enabled computer vision concepts are applied to build a social distancing detector. A system capable of detecting distances between individuals and warning them when distances are not maintained can slow down the spread of the disease (Yang et al. 2020). The detector can be installed in CCTV cameras in public places to monitor if people strictly follow social distancing norms through a program coded to check the function and calculate the distance between two different points in a picture. People who follow social distancing are shown in green boxes while those who do not follow social distancing are shown in red box. An AI-based real-time social distancing detection and warning system with a monocular camera and deep learning-based real-time object detectors finds the best fit to ensure social distancing based on real-world datasets with high accuracy. Wireless technologies along with machine learning, computer vision, thermal screening with ultrasound will provide solutions for symptom prediction, detection and contract tracing for implementing social distancing in full practice (Nguyen et al. 2020; Ulhaq et al. 2020).

\section{Social media}

COVID-19 spread was first predicted by the Canadian startup BlueDot on the basis of AI-based algorithms analyzing the social big data with the Government support (Kim 2020). Subsequently, on 30 January 2020, the Director General, World Health Organization declared the outbreak as an international 
emergency concern and declared it as a pandemic after six weeks (Ienca and Vayena 2020) and collectively called for coordinated structure to support preparedness in response to the fight against the virus across the nations (Reeves et al. 2020). By analyzing the online search pattern of individuals in the affected area provide cues on the priorities of the localized population. On an average 1 billion people per day depend on Baidu search engine to know about COVID-19 related authentic health information (Xu et al. 2020). Initially since Wuhan was the center of this disease, people from Wuhan frequently searched for protective materials, medical help, progress of pandemic, back to school, etc. Some parts of the country searched more on online education while some gave up for online medical health care. The search findings reveal the priorities in health infrastructure and pattern of social panic so that effective prevention and control measures to contain COVID-19 can be deployed.

Countries that have been successful in maintaining low COVID-19 cases and low COVID-19 mortality rates are the ones who detected the virus hotspots in advance along with early tracing and testing of the affected ones. In addition, these countries ensured strict quarantine and social distancing. Efficient coordination and data management with the help of digital technology builds a strong administrative system for disease analysis using big data and to initiate governance mechanisms to mitigate the spread of the virus (Whitelaw et al. 2020). The information regarding the number of people who left Wuhan over a day is obtained from search engines including Baidu and WeChat, which provided valuable data on travel history of persons from Wuhan. Later, ML models used this data to predict the most likely location of where novel coronavirus might arrive next and this might alert authorities to perform the border checks. A study by Kaiyuan Sun and colleagues testifies to the strength of monitoring news reports and social media to help reconstruct the progression of an outbreak and to provide detailed patient-level information and data in the context of a health emergency (Lou et al. 2020). The pandemic made a shift in the minds of people from other health issues to getting affected with the new virus demanding more authentic data and sensible information to be given out which does not create further panic. Also needs to be viewed from a future perspective as well to bring improvements in health literacy and the ability to deal with social havoc. The main motive is to utilize technology and reduce the negative impact associated to minimize the side effects of COVID-19 and social media could play a major role in this (Kuziemski and Misuraca 2020).

Analysis and prediction of upcoming COVID-19 impact with the presently available database from social media platforms using regression models and exponential polynomial is done using machine learning-based modeling which focus on the prediction of COVID-19 outbreak for every succeeding week in India (Yadav 2020). Since the conventional models examine all COVID-19 patients as having the same infection rate, an improved susceptible infected (ISI) model is put forward to roughly calculate the diverse infection rates for analyzing the transmission laws and virus development trends (Zheng et al. 2020). Also, by giving due recognition to the public on a large scale, the natural language processing (NLP) module and the long short term memory (LSTM) network are embedded into the ISI model to build the hybrid AI model for the COVID-19 prediction. This, AI hybrid technology when compared with the conventional technologies exhibited fewer errors in the prediction process (Hsiang et al. 2020). In a way, AI can give early warning systems based on authentic information from trusted sources such as social media, search engines to improve the public policy involvements.

\section{Industry applications}

Next, the role played by the industry is commendable in this mayhem by providing an array of hardware and software products to marginalize the counter-effects of the pandemic. Here, some of the interesting products developed by start-up companies using artificial intelligence to track the pandemic in the larger sphere and proved to be successful are enlisted. One of the noticeable applications of AI during COVID-19 pandemic is BlueDot algorithm using natural language processing (NLP) to skim the text of hundreds of thousands of sources and to scour news and public statements about the health of humans, subsequently giving an early warning on the novel coronavirus outbreak. AI-based computer vision cameras are set up to detect crowds and monitor people during lockdown in China. AI-powered autonomous service robots and humanoid robots called Cloud Ginger were also developed to serve food and 
medicines to patients in hospitals at Wuhan (Dananjayan and Raj 2020). Similarly, a robot Nightingale-19 (Qureshi et al. 2020) is made use of to provide food and medicines to patients at the district coronavirus care center in India where a large number of cases have been reported. When the world finally recovers from the huge spike in COVID-19 cases, robots with efficient AI technology will find applications in the service industry as a whole.

Many notable institutions as well as startups have come up with valuable applications of AI with the current situation in mind so as to predict the probable location of future outbreaks. Huge responses have been coming up in the way of AI applications and it includes BenevolentAI and Imperial College London collaborative project (Stebbing et al. 2020). COVID19 could be detected with AI technology induced cameras equipped with thermal sensors developed by KroniKare, a startup company (Johnson 2020). Infervision, software created by a Beijing-based AI startup company uses its algorithm to spot COVID-19 on images of the lung as distinct from other respiratory infections and has been deployed at 34 hospitals in China to examine over 32000 cases. By close examination of many images of CT scans the software can detect images having lung problems by pattern recognition (Shi et al. 2020). Similarly, an AI startup Closedloop, is using healthcare data to identify patients at the highest risk of severe complications from COVID-19 with an AI-based predictive model that identifies people with high risk of getting the virus.

The San Francisco-based Startup Company Metabiota launched an Epidemic tracking mechanism to inform people and authorities in advance about any outbreaks that might happen in the near future. It is done by assembling data from various places, hospitals and clinics to chart out where there is a possibility of disease (Shieber 2020). In Korea, the development of a kit for rapidly diagnosing the potentially infected and innovative test methods such as drive-through screening centers, enabled thousands of people to be tested every day (Shaw et al. 2020). This large-scale diagnosis for COVID-19 was able to detect and confirm cases in their early stages, thus lowering the fatality rate and preventing the widespread spread of the infectious disease. During the time when COVID19 cases were at the highest in China, February 2020, a Danish company in agreement with the healthcare officials to distribute robots which wander around health care facilities with the help of UV-light to disinfect rooms contaminated with bacteria or viruses (Johnson 2020). Further, by combining computer vision (Ulhaq et al. 2020) and infrared sensor the forehead temperature of 200 people in a minute was identified. Drones are an important device and more than 100 drones were made to be used for spraying disinfectant and thermal sensing by Shenzhen Micro MultiCopter. Also drones helped in patrolling public places and collecting useful data and information for further processing (Johnson 2020). AI is also used to respond to coronavirus with the help of a few other applications such as Corona maps, Corona reminders, and mask reminders in Korea (Kim 2020). For the healthcare sector which has an increasing demand round the clock has to re-sort to machine learning enabled chat-bots for effective contact-less screening of COVID-19 symptomatic people and also to answer people's queries. Clevy.io, a French start-up, has launched a chat-bot to help people find official Government communications about COVID-19. Authorized by real-time information from the French Government and the WHO, the chat-bot evaluates known symptoms and answers queries if any about Government policies. The chat-bot has been successful to answer questions on everything about COVID19 risks, without much pressure on the healthcare and Government institutions. The key findings of the contribution of AI in public sphere disease management are summarized in Table 1.

\section{Limitations and future directions}

Models can reveal principles about outbreaks and determine which interventions can bring down the case numbers effectively. Mathematical models of epidemiological and social modeling apply AI/ML for data assimilation and estimation against covid-19 and simple models like the SIR model can be extensively applied to identify asymptomatic infectious individuals (Thompson 2020). Agent-based modeling is a new approach comprising of agents behavior portrayed with distinct characteristics to present more realistic results where simulated agents make decisions based on the planned rules. The model includes individual profile of each agent with social and health conditions during interactions with other individuals and various 
Table 1 Big data analysis using AI strategies in the public sphere to control the virus spread: a summary of the findings

\begin{tabular}{|c|c|c|}
\hline Public sphere & Domain of interventions & AI and associated strategies \\
\hline $\begin{array}{l}\text { Health } \\
\text { infrastructure }\end{array}$ & $\begin{array}{l}\text { Chatbots, Health barcode system } \\
\text { Screening methods, Electronic health records, disease spread } \\
\text { forecasting }\end{array}$ & $\begin{array}{l}\text { Machine learning, Deep learning } \\
\text { Random-forest, linear and auto-regressions }\end{array}$ \\
\hline Surveillance & $\begin{array}{l}\text { Participatory disease surveillance system, } \\
\text { Aarogya Setu application Ariel and mobile robotics Thermal } \\
\text { imaging }\end{array}$ & $\begin{array}{l}\text { Sentiment Informed Time Series Analysis } \\
\text { National Geographic Information System 5G } \\
\text { and block chain technologies }\end{array}$ \\
\hline Contact tracing & $\begin{array}{l}\text { Baidu big data Aarogya Setu app, } \\
\text { Bluetooth-based proximity tracing system }\end{array}$ & DNN, GPS, real-time big data analysis \\
\hline Social distancing & $\begin{array}{l}\text { Social distancing detector, CCTV cameras, Thermal camera, } \\
\text { ultrasound sensors }\end{array}$ & $\begin{array}{l}\text { Machine learning, Computer vision, } \\
\text { Wireless technologies, }\end{array}$ \\
\hline Social media & Baidu and Google search engines, We-chat & Hybrid AI model \\
\hline $\begin{array}{l}\text { Industrial } \\
\text { applications }\end{array}$ & $\begin{array}{l}\text { BlueDot algorithm, vision cameras, } \\
\text { Mask reminders, Chat-bot, Humanoid robots }\end{array}$ & Machine and Deep learning \\
\hline
\end{tabular}

experimental results demonstrating useful information to construct strategies to reduce the transmission of COVID-19 within the facilities (Cuevas 2020).

Even though AI has shown remarkable development in the case of COVID-19 management there are pitfalls or limitations. Currently, AI techniques are not matured enough to meet the requirements; however in future it is expected to be the game changer and can save many lives and limit damages to the society (Naud'e 2020). AI is still at a preliminary stage, so it takes much time for the results to be notable, moreover among the proposed AI systems very few have operational maturity. Compromise on data privacy and security is one of the major drawbacks of the AI. The public are actually worried that the data collected may be misused for other purposes. Even though the data collected during this pandemic are for controlling the spread of the disease there is a fear in the minds of the public that in the post-Covid scenario the collected data may not be handled properly. Another shortcoming is that with the development of $\mathrm{AI}$ in other areas will put a pressure on the public to adopt the technologies as the digital platforms and digitalization are being innovated day-by-day (Naud'e 2020). Since big data is involved in AI, it is mandatory to share timely and most accurate information across medical, educational, economic and industrial sectors. Since the accurate information cannot be achieved it leads to less effectiveness of AI. Hence different strategies adopted by different countries are analyzed and compared and the best methods are improvised. It is still too early to tell if and to what extent AI will have an impact on the COVID-19 outbreak. Even though AI has huge potential in improvising healthcare facilities with promising results, the inherent data processing strategy is considered as a black box owing to its inability to deduce logical conclusions. In addition, liability issues are a major challenge with conflict of interest from the users' side in utilizing $\mathrm{AI}$ in a complementary fashion. Therefore, for enhanced results, a collaborative effort is needed in developing and optimizing AI strategies using appropriate standard of references as well as unbiased data sets (Teoh et al. 2020).

However, one thing is certain that $\mathrm{AI}$ is relevant to this outbreak and in the future it will become even more critical. A comparative analysis on the merits and demerits of the AI schemes in COVID-19 containment is given in Table 2 .

There were many research works concentrating on AI applications in COVID-19 management in diagnosis and treatment; however the contributions are still limited when it comes to the fight against the outbreak. There can be difficulties in using the applications in the real-world clinical procedures. Future works on machine learning and deep learning models needs to be investigated for transparency and interpret-ability. In an uncertain world, AI can be made use to examine the humongous data to decide on which treatments or procedures would work or should be strived for. The 
Table 2 A summary of the merits and demerits of the AI strategies adopted for the control of COVID-19 disease in public sphere.

\begin{tabular}{|c|c|c|}
\hline AI in social sphere & Merits & Demerits \\
\hline $\begin{array}{l}\text { Health } \\
\text { infrastructure }\end{array}$ & $\begin{array}{l}\text { Faster and better diagnosis than humans } \\
\text { AI-robots reduce risk of transmission Real-time processing of bulk patient } \\
\text { data }\end{array}$ & $\begin{array}{l}\text { Threats of privacy problems, } \\
\text { Ethics concerns, } \\
\text { Diagnosis errors due to biased data }\end{array}$ \\
\hline Surveillance & $\begin{array}{l}\text { Tack and predict disease growth } \\
\text { Interpretation in individual level Fast medical assistance deployment } \\
\text { Thermal imaging }\end{array}$ & $\begin{array}{l}\text { Multiple issues of privacy } \\
\text { Human right issues } \\
\text { Inequality in medical attention }\end{array}$ \\
\hline Contact tracing & $\begin{array}{l}\text { Interruption in transmission chain } \\
\text { Disease spread real-time monitoring }\end{array}$ & $\begin{array}{l}\text { Privacy and trust breach of mobile } \\
\text { apps } \\
\text { Personal data security } \\
\text { Compromise on data } \\
\text { confidentiality }\end{array}$ \\
\hline Social distancing & $\begin{array}{l}\text { Reduction in human contact instances } \\
\text { Improved awareness among citizens }\end{array}$ & $\begin{array}{l}\text { Reduced economic activity } \\
\text { Cascading effect on low-income } \\
\text { people }\end{array}$ \\
\hline Social media & $\begin{array}{l}\text { Quick communication with medical team } \\
\text { Effective sharing of critical information Removal of geographic } \\
\text { boundaries }\end{array}$ & $\begin{array}{l}\text { Dissemination of erroneous } \\
\text { information } \\
\text { Spreading of exaggerated/ } \\
\text { unsolicited info }\end{array}$ \\
\hline $\begin{array}{l}\text { Industrial } \\
\text { applications }\end{array}$ & $\begin{array}{l}\text { Development of deployable technology } \\
\text { Infrastructure built-up for future scenario Development of scalable } \\
\text { solutions }\end{array}$ & $\begin{array}{l}\text { Lack of time for testing and } \\
\text { validation }\end{array}$ \\
\hline
\end{tabular}

major improvements AI brought in during the COVID-19 times itself are promising. When a set of data is provided with the help of AI it helps much in bringing clarity to do the next step. It was with the help of AI technology the new spread of similar symptomatic disease was found. Hence AI can be effectively utilized for predicting and analyzing future outbreaks or diseases. With the advent of AI within the next decade inception to clinical testing may go to just months duration. From journals to databases to clinical trials information is sourced for bringing out much quality to life. To make it all applicable in the near future, AI engineers and developers will have to plan ahead and think before it occurs; such predictions should be made possible with the large pool of data. Furthermore, to ensure the quality data by filtering out the biased data from the generated datasets is required which further will elevate the accuracy and reduce the over-fitting of AI predictions.

\section{Summary}

In this review, various AI strategies and techniques applied in public sphere using big-data analysis to prevent the dissemination of unexpected COVID-19 pandemic are discussed in detail. Figure 2 shows the big data analysis using AI strategies in the public sphere to control the virus spread.

The disease prevention in public sphere is attained by improving health infrastructure, enhancing surveillance, strengthening contact tracing, intensifying social distancing, augmenting social media and upgrading industrial applications through the AI techniques. It is noticed that, the big data generated from chat-bots, digital health records, contact tracing mobile applications, robotic platforms, image and thermal sensors can be used to train efficient and fast AI networks to deduce useful information regarding the impact of virus spread. Moreover, the advanced AI techniques such as machine learning, deep learning and regression models are also modified and applied in various pandemic mitigation measures. This review is expected to show light into the merits and demerits of 


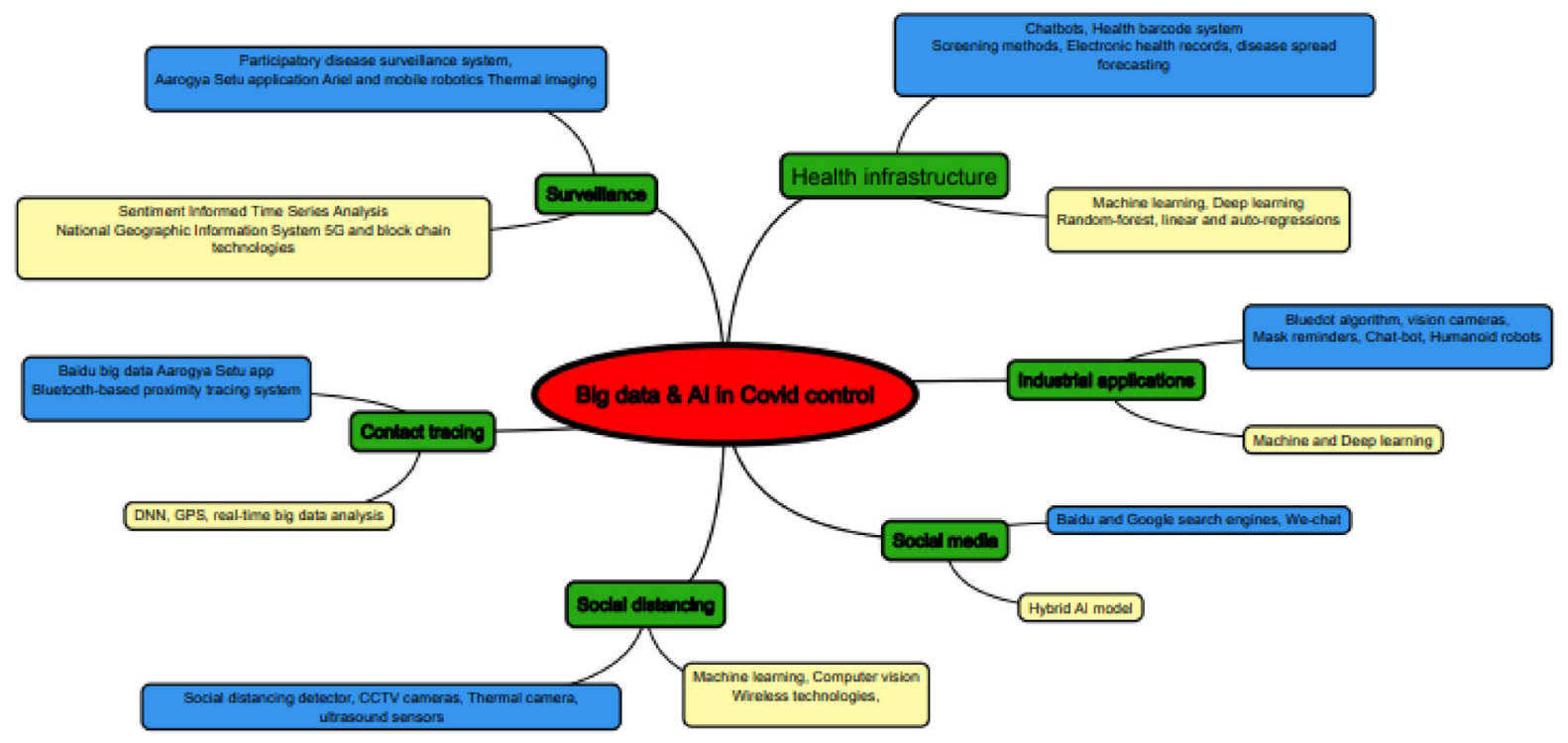

Fig. 2 Contribution of $\mathrm{AI}$ in public sphere disease management

the AI strategies adopted. Furthermore, it is noticed that a biased data set may lead to wrong interpretation and subsequent error in judgment of the COVID-19 scenario. Most importantly, in this digital era, automated decision making on certain specific areas might come as a big relief for the Governments and nongovernmental agencies to concentrate more on the most essential and critical areas connected with pandemic prevention. It is obvious that, in any possible future outbreaks AI will play a more crucial role in the diagnosis and control of pandemic/ epidemic as more and more data is digitized every day.

Funding No funding received.

\section{Declaration}

Conflict interests The authors declare that there is no potential conflicts of interest.

\section{References}

Ahuja AS, Reddy VP, Marques O (2020) Artificial intelligence and COVID-19: a multidisciplinary approach

Allam Z, Jones DS (2020) On the coronavirus (covid-19) outbreak and the smart city network: universal data sharing standards coupled with artificial intelligence (AI) to benefit urban health monitoring and management. In: Healthcare, vol 8, p 46. Multidisciplinary Digital Publishing Institute
Al-Timemy AH, Khushaba RN, Mosa ZM, Escudero J (2020) An efficient mixture of deep and machine learning models for covid-19 and tuberculosis detection using X-ray images in resource limited settings. arXiv preprint arXiv:2007.08223

Banerjee A, Ray S, Vorselaars B, Kitson J, Mamalakis M, Weeks S, Baker M, Mackenzie LS (2020) Use of machine learning and artificial intelli- gence to predict sars-cov-2 infection from full blood counts in a population. Int Immunopharmacol 106705

Bay J, Kek J, Tan A, Hau CS, Yongquan L, Tan J, Quy TA (2020) BlueTrace: a privacy-preserving protocol for community-driven contact tracing across borders. Government Technol Agency Singapore Tech Rep

Beard S, Belchamber J (2020) AI and data governance issues in responding to covid-19: a briefing

Bobdey S, Ray S et al (2020) Going viral-covid-19 impact assessment: a perspective beyond clinical practice. J Mar Med Soc 22(1):9

Bullock J, Pham KH, Lam CS, Luengo-Oroz $\mathrm{M}$ et al (2020)Mapping the landscape of artificial intelligence applications against covid-19. arXiv preprint arXiv:2003.11336

Chamola Vinay, Hassija Vikas, Gupta Vatsal, Guizani Mohsen (2020) A comprehensive review of the covid-19 pandemic and the role of IoT, drones, AI, blockchain, and $5 \mathrm{G}$ in managing its impact. IEEE Access 8:90225-90265

Chen J, See KC (2020) Artificial intelligence for covid-19: rapid review. J Med Internet Res 22(10):e21476

Courtemanche C, Garuccio J, Le A, Pinkston J, Yelowitz A (2020) Strong social distancing measures in the united states reduced the covid-19 growth rate: study evaluates the impact of social distancing measures on the growth rate of confirmed covid-19 cases across the united states. Health Aff 39(7):1237-1246 
Cuevas E (2020) An agent-based model to evaluate the COVID19 transmission risks in facilities. Comput Biol Med 121:103827. https://doi.org/10.1016/j.compbiomed.2020. 103827

Dananjayan S, Raj GM (2020) Artificial intelligence during a pandemic: the covid-19 example. Int J Health Plan Manag

Desai PS (2002) Sentiment informed timeseries analyzing ai (sitala) to curb the spread of covid-19 in houston. medRxiv

Dhamodharavadhani S, Rathipriya R, Chatterjee JM (2020) COVID-19 mortality rate prediction for India using statistical neural network models. Front Public Health 8

Dwivedi YK, Hughes DL, Coombs C, Constantiou I, Duan Y, Edwards JS, Gupta B, Lal B, Misra S, Prashant P et al (2020) Impact of covid-19 pandemic on information management research and practice: transforming education, work and life. Int J Inf Manag 55:102211

Emanuel EJ, Wachter RM (2019) Artificial intelligence in health care: will the value match the hype? Jama 321(23):2281-2282

Fang R, Xiao Y, Tian J, Pouyanfar S, Yang Y, Chen SC, Iyengar SS (2019) Big data in computational health informatics. Big Data Multimodal Med Imaging 103

Garg S, Bhatnagar N, Gangadharan N (2020) A case for participatory disease surveillance of the covid-19 pandemic in india. JMIR Public Health Surveill 6(2):e18795

Garofalo G, Preuveneers D, Joosen W, Abidin A, Mustafa MA (2020) Striking the balance: effective yet privacy friendly contact tracing. IACR Cryptol ePrint Arch 2020: 559

Golinelli D, Boetto E, Carullo G, Nuzzolese AG, Landini MP, Fantini MP (2020) How the COVID-19 pandemic is favoring the adoption of digital technologies in healthcare: a literature review. medRxiv

He J, Baxter SL, Xu J, Xu J, Zhou X, Zhang K (2019) The practical implementation of artificial intelligence technologies in medicine. Nat Med 25(1):30-36

Hossain MS, Muhammad G, Guizani N (2020) Explainable AI and mass surveillance system-based healthcare framework to combat covid-i9 like pandemics. IEEE Netw 34(4):126-132

Hsiang S, Allen D, Annan-Phan S, Bell K, Bolliger I, Chong T, Druckenmiller H, Huang LY, Hultgren A, Krasovich E et al (2020) The effect of large-scale anti-contagion policies on the COVID-19 pandemic. MedRxiv

Hsieh P (2017) Ai in medicine: rise of the machines. Forbes. Forbes Magazine 30

$\mathrm{Hu}$ Z, Ge Q, Li S, Jin L, Xiong M (2020) Artificial intelligence forecasting of covid-19 in china. arXiv preprint arXiv:2002.07112

Hui DS, Azhar EI, Madani TA, Ntoumi F, Kock R, Dar O, Ippolito G, Mchugh TD, Memish ZA, Drosten C et al (2020) The continuing 2019-nCoV epidemic threat of novel coronaviruses to global health-The latest 2019 novel coronavirus outbreak in Wuhan, China. Int J Infect Dis 91:264-266

Ienca M, Vayena E (2020) On the responsible use of digital data to tackle the covid-19 pandemic. Nat Med 26(4):463-464

Iwendi C, Bashir AK, Peshkar A, Sujatha R, Chatterjee JM, Pasupuleti S, Mishra R, Pillai S, Jo O (2020) Covid-19 patient health prediction using boosted random forest algorithm. Front Public Health 8:357
Jakhar D, Kaur I (2020) Current applications of artificial intelligence for covid-19. Dermatol Ther

Jamal DN, Rajkumar S, Ameen N (2018) Remote elderly health monitoring system using cloud-based wbans. In: Handbook of research on cloud and fog computing infrastructures for data science, pp 265-288. IGI Global

Johnson K (2020) How people are using ai to detect and fight the coronavirus, the machine. https://venturebeat.com/2020/ 03/03/how-people-are-using-ai-to-detect-and-fight-thecoronavirus/

Kim H (2020) The response of AI systems in other countries to corona virus (covid-19) infections: E-government, policy, AI utilizing cases. J Digit Converg 18(6):479-493

Kumar A, Sharma GK (2020) Artificial intelligence technologies combating against COVID-19. Dev Sanskriti Interdiscip Int J 16:56-60

Kuziemski M, Misuraca G (2020) AI governance in the public sector: three tales from the frontiers of automated decisionmaking in democratic settings. Telecommun Policy 44:101976

Lalmuanawma S, Hussain J, Chhakchhuak L (2020) Applications of machine learning and artificial intelligence for covid-19 (sars-cov-2) pandemic: a review. Chaos Solitons Fractals 110059

Lardinois F (2020) IBM, Amazon, Google and Microsoft partner with White House to provide compute resourcesfor covid-19 research

Leslie D (2020) Tackling covid-19 through responsible AI innovation: five steps in the right direction. Harv Data Sci Rev

Li LM, Ayscue P (2020) Using viral genomics to estimate undetected infections and extent of superspreading events for covid-19. medRxiv

Liu X, Rivera SC, Moher D, Calvert MJ, Denniston AK (2020) Reporting guidelines for clinical trial reports for interventions involving artificial intelligence: the consort-ai extension. bmj 370

Lou J, Tian SJ, Niu SM, Kang XQ, Lian HX, Zhang LX, Zhang JJ (2020) Coronavirus disease 2019: a bibliometric analysis and review. Eur Rev Med Pharmacol Sci 24(6):3411-21

Luengo-Oroz M, Pham KH, Bullock J, Kirkpatrick R, Luccioni A, Rubel S, Wachholz C, Chakchouk M, Biggs P, Nguyen T et al (2020) Artificial intelligence cooperation to support the global response to covid-19. Nat Mach Intell 1-3

Maniou TA, Veglis A (2020) Employing a chatbot for news dissemination during crisis: design, implementation and evaluation. Future Internet 12(7):109

McCall Becky (2020) Covid-19 and artificial intelligence: protecting health-care workers and curbing the spread. The Lancet Digital Health 2(4):e166-e167

Mohamadou Y, Halidou A, Kapen PT (2020) A review of mathematical modeling, artificial intelligence and datasets used in the study, prediction and management of covid-19. Appl Intell 50(11):3913-3925

Mohanty S, Rashid MH, Mridul M, Mohanty C, Swayamsiddha S (2020) Application of Artificial Intelligence in COVID19 drug repurposing. Diabet Metab Syndr Clin Res Rev

Naudé W (2020) Artificial intelligence Vs Covid-19: limitations, constraints and pitfalls. Ai and Society, $\mathrm{p} 1$

Nguyen CT, Saputra YM, Van Huynh N, Nguyen NT, Khoa TV, Tuan BM, Nguyen DN, Hoang DT, Vu TX, Dutkiewicz E 
et al (2020) Enabling and emerging technologies for social distancing: A comprehensive survey. arXiv preprint arXiv:2005.02816

Pappot N, Taarnhøj GA, Pappot H (2020) Telemedicine and e-health solutions for covid-19: patients' perspective. Telemed e Health

Pearl R (2018) Artificial intelligence in healthcare: separating reality from hype

Punn NS, Sonbhadra SK, Agarwal S (2020) COVID-19 epidemic analysis using machine learning and deep learning algorithms. medRxiv

dQureshi D, Salim M, Singh P, Swarnkar P, Goud H (2020) Robotics solutions to combat novel corona virus disease2019 (covid-19). Pushpendra and Swarnkar, Pankaj and Goud, Harsh, Robotics Solutions to Combat Novel Corona Virus Disease-2019 (COVID-19) (June 2, 2020)

Ranga V, Pani P, Kanga S, Meraj G, Farooq M, Nathawat MS, Singh SK (2020) National health-gis portal-a conceptual frame- work for effective epidemic management and control in India

Raza K (2020) Artificial intelligence against COVID-19: a meta-analysis of current research. Big Data Anal ArtifIntell COVID-19 Innov Vis Approach 165-176

Reeves JJ, Hollandsworth HM, Torriani FJ, Taplitz R, Abeles S, Tai-Seale M, Millen M, Clay BJ, Longhurst CA (2020) Rapid response to covid-19: health informatics support for outbreak management in an academic health system. J Am Med Inf Assoc 27(6):853-859

Reps J, Kim C, Williams R, Markus A, Yang C, Salles TD, Falconer T, Jonnagaddala J et al (2002) Can we trust the prediction model? Demonstrating the importance of external validation by investigating the covid-19 vulnerability (c-19) index across an international network of observational healthcare datasets. medRxiv

Rivera SC, Liu X, Chan AW, Denniston AK, Calvert MJ (2020) Guidelines for clinical trial protocols for interventions involving artificial intelligence: the spirit-ai extension. bmj 370

Schwartz JT, Gao M, Geng EA, Mody KS, Mikhail CM, Cho SK (2019) Applications of machine learning using electronic medical records in spine surgery. Neurospine 16(4):643

Shaw R, Kim YK, Hua J (2020) Governance, technology and citizen behavior in pandemic: lessons from covid-19 in East Asia. Prog Disaster Sci 100090

Shi F, Wang J, Shi J, Wu Z, Wang Q, Tang Z, He K, Shi Y, Shen D (2020) Review of artificial intelligence techniques in imaging data acquisition, segmentation and diagnosis for covid-19. IEEE Rev Biomed Eng

Shieber J (2020) World economic forum. http://www.mattoxaz. com/category/world-\%20economic-forum/page/2/

Shuja J, Alanazi E, Alasmary W, Alashaikh A (2020) Covid-19 open source data sets: a comprehensive survey. medRxiv

Simonite T (2020) Chinese hospitals deploy ai to help diagnose covid-19. Wired 26
Stebbing J, Phelan A, Griffin I, Tucker C, Oechsle O, Smith D, Richardson P (2020) Covid-19: combining antiviral and anti-inflammatory treatments. Lancet Infect Dis 20(4):400-402

Sujath R, Chatterjee JM, Hassanien AE (2020) A machine learning fore- casting model for covid-19 pandemic in india. Stoch Environ Res Risk Assess 1

Taylor EJ (2020) AI and global governance: covid-19 underlines the need for AI- support governanceof water-energyfood nexus, AI and global governancec

Teoh JY, Chiong E, Ng CF (2020) Can artificial intelligence optimize case selection for hemi-gland ablation?. BJU Int 125(3): 333-334

Thompson RN (2020) Epidemiological models are important tools for guiding COVID-19 interventions. BMC Med 18:152. https://doi.org/10.1186/s12916-020-01628-4

Ulhaq A, Khan A, Gomes D, Paul M (2020) Computer vision for covid-19 control: a survey. arXiv preprint arXiv:2004.09420

Vaishya R, Javaid M, Khan IH, Haleem A (2020) Artificial intelligence (AI) applications for covid-19 pandemic. Diabet Metab Syndr Clin Res Rev

Whitelaw S, Mamas MA, Topol E, Van Spall HG (2002) Applications of digital technology in covid-19 pandemic planning and response. Lancet Digit Health

Wong SH, Teoh JY, Leung CH, Wu WK, Yip BH, Wong MC, Hui DS (2020) Covid-19 and public interest in face mask use. Am J Respir Crit Care Med 202(3):453-455

Wottawa CR, Genovese B, Nowroozi BN, Hart SD, Bisley JW, Grundfest WS, Dutson EP (2016) Evaluating tactile feedback in robotic surgery for potential clinical application using an animal model. Surg Endosc 30(8):3198-3209

Xu C, Zhang X, Wang Y (2020) Mapping of health literacy and social panic via web search data during the covid-19 public health emergency: infodemiological study. J Med Internet Res 22(7):e18831

Yadav RS (2020) Data analysis of covid-2019 epidemic using machine learning methods: a case study of india. Int J InfTechnol 1-10

Yan T, Wong PK, Ren H, Wang H, Wang J, Li Y (2020) Automatic distinction between covid-19 and common pneumonia using multi-scale convo- lutional neural network on chest ct scans. Chaos Solitons Fractals 110153

Yang D, Yurtsever E, Renganathan V, Redmill KA, Özgüner Ü (2020) A vision-based social distance and critical density detection system for covid-19. arXiv preprint arXiv:2007.03578

Zheng N, Du S, Wang J, Zhang H, Cui W, Kang Z, Yang T, Lou B, Chi Y, Long H et al (2020) Predicting covid-19 in china using hybrid ai model. IEEE Trans Cybern

Publisher's Note Springer Nature remains neutral with regard to jurisdictional claims in published maps and institutional affiliations. 